\title{
Revisiting Van Valen's Red Queen Hypothesis
}

\author{
Ricard Solé1, 2, 3 \\ ${ }^{1}$ ICREA-Complex Systems Lab, Universitat Pompeu Fabra (GRIB), Dr Aiguader 80, 08003 Barcelona. \\ ${ }^{2}$ Institut de Biologia Evolutiva, CSIC-UPF, Pg Maritim de la Barceloneta 37, 08003 Barcelona. \\ ${ }^{3}$ Santa Fe Institute, 1399 Hyde Park Road, Santa Fe NM 87501, USA.
}

\begin{abstract}
Leigh Van Valen was an American evolutionary biologist who made major contributions to evolutionary theory and is particularly remembered by his groundbreaking paper "A New Evolutionary Law" (1973) where he provided evidence from fossil record data that this law maintains that the probability of extinction within any group remains essentially constant through time. In order to explain such unexpected result, Van Valen formulated a very influential idea that he dubbed the "Red Queen hypothesis". It states that the constant decay must be a consequence of evolutionary interactions among connected species within ecological networks. In Van Valen's picture, species do not merely evolve: they also coevolve with other species. As a consequence, when thinking in adaptation to an external environment, the other species must be considered as part (may be a major part) of such external world. Van Valen's law provided the first complex systems theory of coevolutionary dynamics and inspired a whole range of theoretical and experimental developments and scholars from very diverse fields, from economics to physics. In that respect, Leigh Van Valen's contribution percolated far beyond its original formulation. Red Queen arms races are nowadays considered a widespread feature of complex adaptive systems.
\end{abstract}

Keywords: Complexity, population dynamics, Red Queen, scales, coevolution, extinction

\begin{abstract}
Now, here, you see, it takes all the running you can do, to keep in the same place.
\end{abstract}

Through the looking glass. Lewis Carroll.

I remember well one day, as an undergraduate student, hearing someone talking about a theory of evolution that was somewhat inspired in a conversation between Alice and the Red Queen in Through the Looking Glass [12]. It was something about ecosystems as a whole as an intrinsic part of the "environment" of each species. I was already interested in complexity and the concept that the whole was more than the sum of the parts. I eventually found a copy of the 1973 typewrited article: "A new evolutionary law" [53] not elegantly edited as most top journals but instead looking like home made (or as paleobiologist David Jablonski puts it, having an "incidental esthetics"). The paper involved two parts, both fascinating.

The first dealt with evidence from fossil record data of a seemingly universal "law of constant extinction": for every example studied (with exceptions, as it always occurs in biology) the members of a given phylogenetic group, from Foraminifera to Ammonoidea to reptiles and mammals, appeared to get extinct with essentially the same probability, as if their actual life length were not relevant at all. In the second part, Van Valen suggested that one way of interpreting the constant extinction could be a "Red Queen dynamics" scenario, where all species change while experiencing "mutually incompatible optima within an adaptive zone. A self-perpetuating fluctuation results which can be stated in terms of an unstudied aspect of zero-sum game theory ${ }^{1}$ ". In other words, each species tries to improve its fitness but in doing so it modifies the evolutionary response of its partners in the ecological network which then also change triggering further responses in the former.

This was in fact a higher-level narrative of the coevolutionary dynamics picture: a prey becomes more cryptic or faster and as a consequence its predator needs to improve its sight or speed. Each of these changes occurs in a context where cost and trade-offs are present. Not all changes are possible or limitless. But when many species are involved, a much more complex situation arises, where each one responds to (and affects) the changes in the others i. e. the "biotic environment". The consequence would be a rather unexpected one: everyone needs to keep changing to remain in the (evolutionary) game. In that perspective, a reductionist view of ecosystems as sets of species whose fate would depend on environmental changes was replaced by a very different one with extinction being the result of intrinsic, system-level changes.

I was not aware then that the would-be enormously influential paper had been rejected in several leading journals, including Nature. That chain of rejections led to the creation, by Van Valen himself, of a new journal, Evolutionary Theory, the first article being no other than the constant extinction and the Red Queen hypothesis. Despite its difficult beginnings, the article started to become very well known, eventually generating a major response.

\footnotetext{
1 In game theory, a zero-sum game is a mathematical representation of a scenario where each participant's gain or loss is exactly balanced by the losses or gains of the other participants.
} 

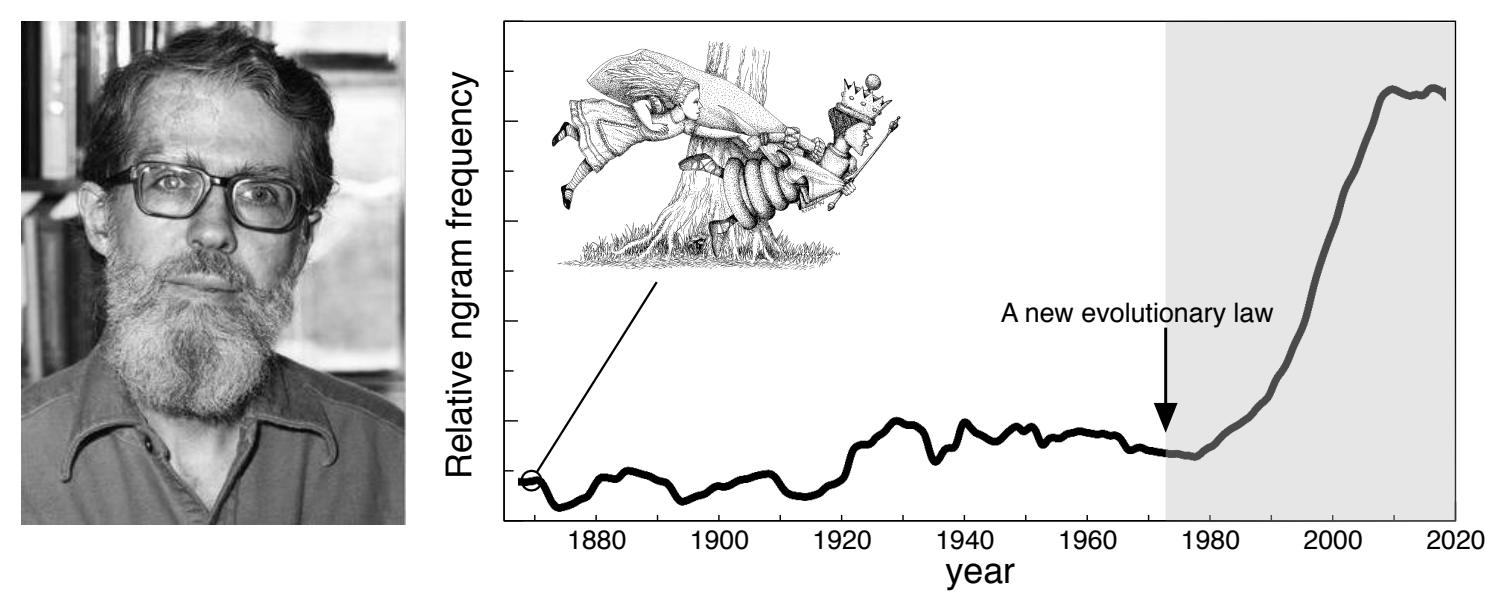

FIG. 1 Van Valen and the rise of the Red Queen. The impact of Van Valen's Red Queen hypothesis can be appreciated in the right panel, where the Google ngrams viewer tool (https://books.google.com/ngrams) has been used to measure the relative frequency of the term "Red Queen" within a very large corpus of written texts. The plot marks the publication of Lewis Carroll's Through the looking glass (drawing by R. Solé) and the publication of van Valen's classic paper where his theory was first introduced. A rapid growth of citations is noticeable, indicating a marked spread of the concept within the scientific literature.

Nowadays, it considered by many as one of the most influential papers in evolutionary biology of the 20th century, its importance has not faded since. A visual picture of this success is provided by figure 1, where we display the time series of citations of the term "Red Queen" as found in a collection of millions of books. The software detects the number of citations per year and two landmark date are highlighted: the publication of Lewis Carrol's book Through the looking glass (the follow up of Alice in Wonderland) and the publication year of Van Valen's paper. Although Carroll's book was famous in 1973, we can see a marked increase of citations in the aftermath's of "A New Evolutionary Law" that have to do with its scientific relevance.

The idea of a constantly changing, coevolving ecological theatre spread fast and all over the place. It became quickly adopted and tested by evolutionary biologists, researchers in prey-predator or host-pathogen interactions and eventually inspired a plethora of mathematical and computer models [11]. Scholars within economics and sociology used game theory to analyze the problem and physicists developed new theories of changing fitness landscapes. The Red Queen was reigning in the kingdom of evolutionary biology and beyond.

\section{THE CONSTANT EXTINCTION PATTERN}

Van Valen observed that the vast majority of taxonomic groups analyzed displayed exponentially decaying survivorship curves. This result implied constancy in the probability of extinction of the taxa, regardless of their previous duration. That is, both data from the fossil record and from extant species suggested that a given species may disappear at any time, irrespective of how long has already existed. This unexpected phenomenon (the Law of Constant Extinction), can be formulated in a simple way, as follows. If $N(t)$ indicates the number of species at a given time and we follow their presence over time (ignoring other events) we would observe an exponential decay law, namely:

$$
\frac{d N}{d t}=-\delta(t) N
$$

where $\delta(t)$ indicates a time-dependent extinction rate. If $N_{0}$ is the original cohort size, this differential equation is easily solved, and gives:

$$
N(t)=N_{0} \exp \left(-\int_{0}^{t} \delta(t) d t\right)
$$

Despite the seemingly obvious assumption that $\delta$ depends on $t$, the surprising observation is that the observed curves fit very well a constant decay rate $\delta$, i.e., a solution:

$$
N(t)=N_{0} e^{-\delta t}
$$

where $\delta$ is the extinction probability of a species (per millions of years, Myr). A median longevity time $\tau$ can be estimated from this equation, defined as the time required to halve the initial cohort size, I. e. $N(\tau)=N_{0} / 2$. It is easy to show that this time is just

$$
\tau=\frac{\operatorname{Ln} 2}{\delta}
$$

that can be considered a law of Paleobiology [35]. The correctness of this law was soon questioned and became a controversial issue [34]. But for some well-preserved taxa, a constant extinction rate seems consistent with 


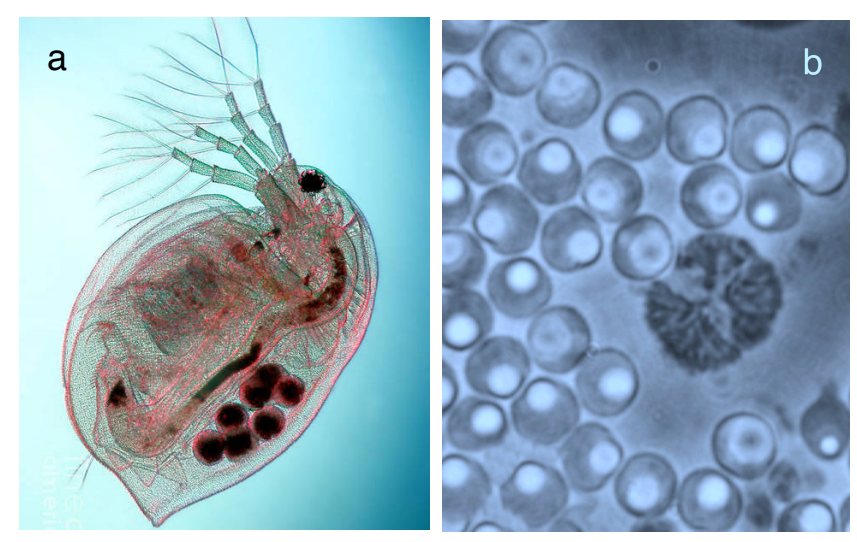

FIG. 2 Experimental systems displaying evolutionary arms races include for example the host-parasite system formed by Daphnia magna (a, image by Ted Kinsman) and its pathogen (b) Pasteuria ramosa, here shown in two different stages in its life cycle (image by Dieter Ebert).

data $[6,7]$. In particular, dedicated studies revealed that species of modern mammals are likely to become extinct as were their ancestors living 200 Myr ago [7]. If evolution leads to improvement through adaptation, why modern mammals have the same extinction probabilities as their ancestors? Here comes the Red Queen.

\section{NEVER-ENDING COEVOLUTION}

The fossil record offers a description of biological change that is rich and revealing: we know that around $99 \%$ of all species that ever lived are now extinct [44]. Moreover, external events are known to play a role in driving large extinction events. In this context, the global pattern of decay of taxonomic cohorts is much more episodic, marked by sharp decays associated to five major extinction events [44? ]. But despite these abrupt, coherent events, the background extinction among major decays suggests a steady extinction. In Van Valen's paper, the explanation for this background extinction is a highly dynamical, but internal phenomenon involving the simultaneous coevolution of many species within communities. Coevolution pervades evolutionary change on multiple scales and It is not exaggerated to say, to paraphrase Dobzhansky's statement, that nothing makes sense in biology except in the light of coevolution. Darwin himself recognized this when referring to what he called the entangled bank [14]:

"It is interesting to contemplate an entangled bank, clothed with many plants of many kinds, with birds singing on the bushes, with various insects flitting about, and with worms crawling through the damp earth".

Indeed, ecosystems need to be seen as collectives of interacting species whose evolutionary fate is necessar- ily intermingled in complex ways [? ]. Van Valen's Red Queen hypothesis suggests that the constant change of the nodes of these networks propagates through the web as other species also respond by evolving themselves. Since change might not happen indefinitely (due to biological constraints) a species unable to cope with change will disappear. Such hypothesis was mathematically formulated by Stenseth and Maynard Smith by means of a simple, two-dimensional model representing two variables: the average lag of different species and the number of species [51]. These authors showed that a constantly changing regime can be possible under the absence of environmental stress. It was also the beginning of a new generation of models mixing both ecological and evolutionary dynamics that has been under development since then. But as well s living entities evolve, so do the theories.

What is the relative importance of biotic versus abiotic components in driving evolution? This is of course the key question and the final answer might be dependent on the scales at which we watch the system. Proving the theory from paleontological data is far from trivial. Research on Cenozoic terrestrial mammals [41] strongly support RQH over environmental-driven causes. This might also apply to other cases studies including dinosaur evolution [5] but in other studies, a mixture of both internal and environmental factors are revealed. What is then the role of each component? Anthony Barnosky suggested a unifying picture where both biotic and abiotic components are included [4]. Under this Court Jester model, "evolution, speciation, and extinction rarely happen except in response to unpredictable changes in the physical environment, recalling the capricious behavior of the licensed fool of Medieval times" [8]. This more general theory (not yet truly formalized) predicts that the ecoevolutionary factors defining the Red Queen dynamics would surely dominate evolution over short time periods, whereas multiple factors such as climate variability or tectonic dynamics would dominate larger scales both in time and space.

The possibility that the Red Queen reigns in the shorter time scales can actually been tested empirically $[11,34]$. Many different case studies confirm the presence of this dynamical regime. A wide range of both theoretical models and microcosm experiments as well as the study of intrahost dynamics of pathogens include, among others: (a) transfer experiments of RNA viruses infecting mammalian cells $[13,48]$. (b) coevolving pathogens and their hosts $[1,28,31,39]$ or (c) in the coevolution of Daphnia magna (Fig. 2a) and its parasitic bacterium Pasteuria ramosa (Fig 2b) [15, 23]. The later is a specially nice example that allowed to prove the consistency of the observed host-parasite dynamics with negative frequency dependent selection, as predicted by RQH. 


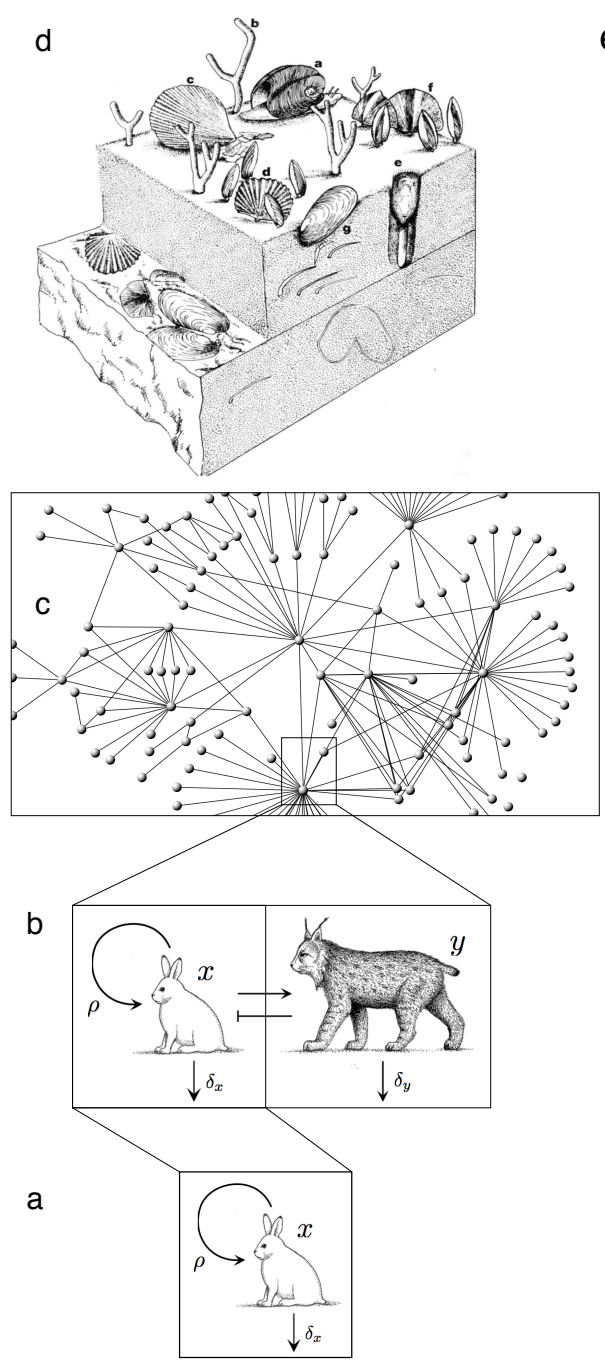

e

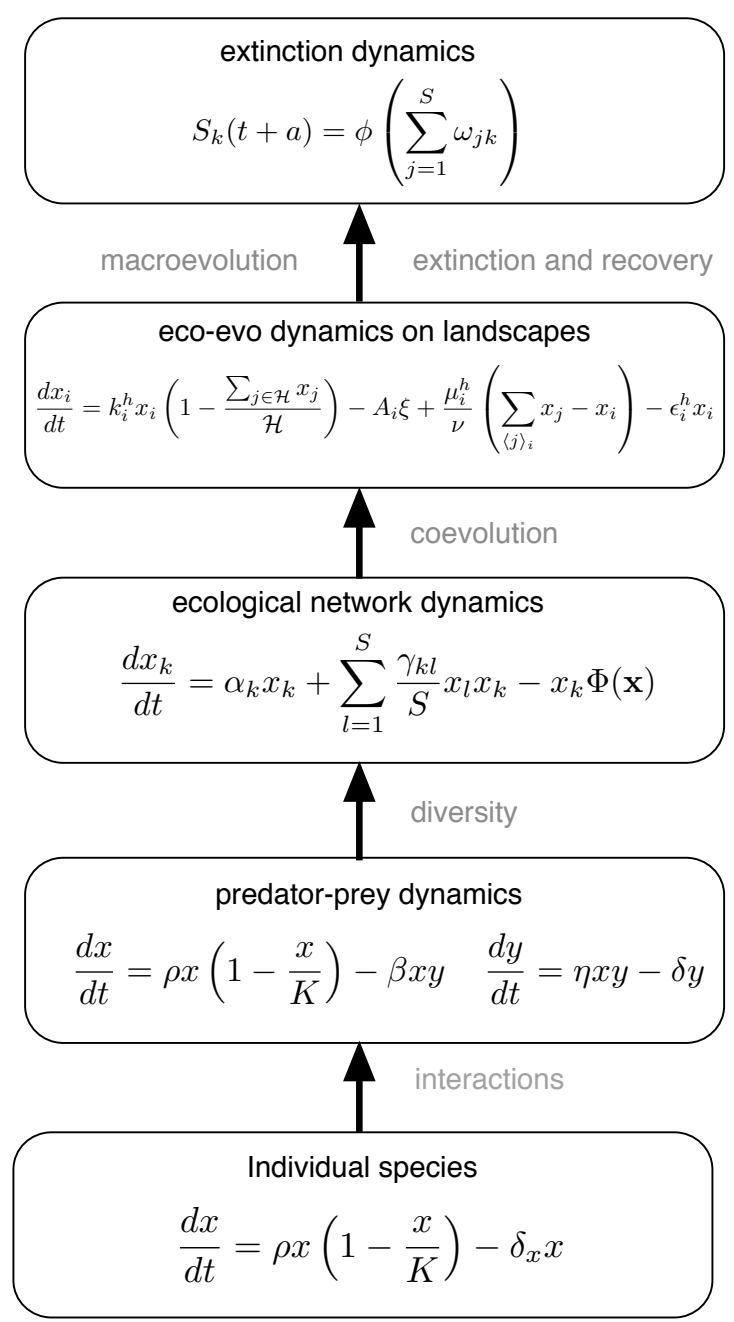

f

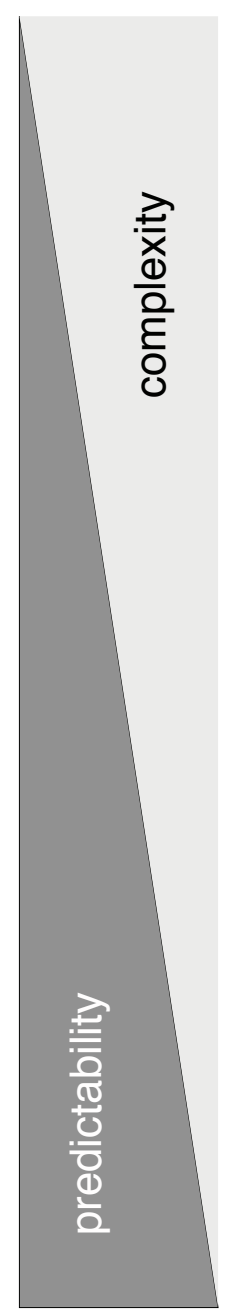

FIG. 3 The multiple scales of ecological and evolutionary change. Here, different levels of complexity involve new phenomena that cannot be reduced to the previous scale. Here we show: (a) single species, (b) pairwise interactions, (c) ecological networks and (d) evolving communities on a macro ecological time scale. Despite the dominant species-level picture of evolution grounded in gene frequencies, the addition of interactions (even pairwise) leads to novel phenomena. Each scale has been explored by means of a diverse range of mathematical models (e): from bottom to top, novel features are included such as species-species interactions, diversity, evolving interactions or large-scale macroevolution. These increasing complexity inevitably leads to increasing complexity and unpredictability as network-level traits become dominant.

\section{EVOLVING NETWORKS}

An undeniable outcome of the RQH has been its inspirational impact for theoretical developments on coevolutionary dynamics and in particular with the use of fitness landscapes as a natural way of coupling coevolutionary interactions [50]. The range of models spans multiple scales (figure 3 ) from pairwise interacting systems to macroevolutionary dynamics. With the rise of Network Science since the early 2000s, the old systems tradition of ecology (particularly within theoretical ecology) has been enriched with the use of both graph theory tools and statistical physics approaches $[20,38]$. In particular, models of large-scale evolution and extinction have shown that the law of constant extinction might be a byproduct of a tendency of coevolving networks to an unstable state [49].

The diverse theoretical developments of Van Valen's picture have flourished in many directions, including (a) the early game theoretic models [51], (b) predator-prey and host-parasite evolution [30, 32, 36, 43, 55], (c) microbiota dynamics [10], (d) the evolution of plant shapes in a virtual environment [24, 26], (e) coevolution of robots [40] and (f) dynamical complexity in economics [3, 45]. It also inspired a new generation of statistical physics models of evolution [20,38]. One of the main triggers for the later has to be found in a landmark paper by Stuart Kauffman and Sonke Johnsen on "Coevolution 
at the edge of chaos" $[2,33]$. The model presented by these authors proposed a well-defined framework to describe Red Queen dynamics in terms of coupled species equipped with a simple "genome" represented as strings of bits. Each of these genomes have an associated fitness measure and mutations push each species up within their fitness landscapes. However, instead of the original conception of fitness landscape as a species-specific structure, the landscapes of different species are coupled between them in such a way that one change in a given landscape can propagate into the others. Under this general framework, RQ dynamics as well as punctuated equilibrium [25] would be an inevitable result of a tendency to critical states $[2,27]$ where ecosystem-level coevolution will led to unpredictable dynamics, effectively decoupling micro- from macroevolution [49].

The Red Queen has been also inspiring further evolutionary metaphors, including (a) the Red King dynamics of mutualistic communities, where the slowly evolving species is likely to gain a disproportionate share of the benefits (instead of the faster changing) [9], (b) the Black Queen Hypothesis, that proposes that gene loss can provide a selective advantage by conserving an organism?s limiting resources [37] and specially relevant for microbial communities or (c) the Suicide King, a phenomenon related to the maladaptive coevolution of parasites that can led the extinction of their hosts [22].

Van Valen had a wide range of interests, spanning the history of all life forms. He had a mind for "out of the box" ideas, such as the concept of cancer as a biological species [54] that has inspired others to re-consider the nature of cancer populations under a different view [21]. "I don't work linearly," he explained in a note to his department chair. "I am a generalist and tend to open new approaches more than fill them in. What I work on changes irregularly and unpredictably with the progress of theory and knowledge." His RQT was certainly a nonlinear, extraordinary insight. The key concept has been shown to be correct and central to our understanding of evolution [18]. In many ways, Van Valen can be said to have been able to look through the Looking Glass and foresee a whole land of open-ended possibilities.

\section{Acknowledgments}

The author thanks Susanna Manrubia, Stuart Kauffman, Santiago Elena, Jose Montoya, Michael Benton, Douglas Erwin and the late Per Bak for useful discussions on fitness landscapes, coevolution and extinction. Special thanks to Josep Sardanyes, for keep chasing the Red Queen with me all these years. This work has been funded by the Botín Foundation and by the Santa Fe Institute.

\section{References}

[1] Agrawal, A., Lively, C. M. Infection genetics: gene-forgene versus matching-alleles models and all points in between. Evol. Ecol. Res. 4, 79-90 (2002)

[2] Bak, P., Flyvjerg, H., Lautrup, B. Coevolution in a Rugged Fitness Landscape. Phys. Rev. A 46, 6724-6730 (1992)

[3] Barnett, W.P. and Hansen, M.T., 1996. The red queen in organizational evolution. Strategic management journal 17, 139-157.

[4] Barnosky, A.D., 2001. Distinguishing the effects of the Red Queen and Court Jester on Miocene mammal evolution in the northern Rocky Mountains. Journal of Vertebrate Paleontology 21, 172-185.

[5] Benson, R.B., Campione, N.E., Carrano, M.T., Mannion, P.D., Sullivan, C., Upchurch, P. and Evans, D.C., 2014. Rates of dinosaur body mass evolution indicate 170 million years of sustained ecological innovation on the avian stem lineage. PLoS Biol. 12, p.e1001853.

[6] Benton, M.J., 1987. Progress and competition in macroevolution. Biol- Rev. 62, 305-338.

[7] Benton, M. J. Red Queen hypothesis. In: Paleobiology, eds. D.E.G. Briggs and P.R. Growther, Blackwell: Oxford (1995)

[8] Benton, M.J., 2009. The Red Queen and the Court Jester: species diversity and the role of biotic and abiotic factors through time. Science 323, 728-732.

[9] Bergstrom, C.T. and Lachmann, M., 2003. The Red King effect: when the slowest runner wins the coevolutionary race. Proc. Natl. Acad. Sci. U.S.A. 100, 593-598.

[10] Bonachela, J.A., Wortel, M.T. and Stenseth, N.C., 2017. Eco-evolutionary Red Queen dynamics regulate biodiversity in a metabolite-driven microbial system. Scientific Reports 7, 1-9.

[11] Brockhurst, M.A., Chapman, T., King, K.C., Mank, J.E., Paterson, S. and Hurst, G.D., 2014. Running with the Red Queen: the role of biotic conflicts in evolution. Proc. R. Soc. B 281, 20141382.

[12] Carroll, L., 1999. The annotated Alice: The definitive edition. WW Norton and Company.

[13] Clarke, D. K., Duarte, E. A., Elena, S. F., Moya, A., Domingo, E., Holland, J. J. The Red Queen reigns in the kingdom of RNA viruses. Proc. Natl. Acad. Sci. U.S.A. 91, 4821-4824 (1994)

[14] Darwin, C. On the Origin of Species by Means of Natural Selection, or the Preservation of Favoured Races in the Struggle for Life, London: John Murray (1859)

[15] Decaestecker, E., Gaba, S., Raeymaekers, J. A. M., Stoks, R., Van Kerckhoven, Ebert, D., Meester, L. D. Host-parasite 'Red Queen' dynamics archived in pond sediment. Nature 450, 870-873 (2007)

[16] Dercole, F., Ferriere, R., Rinaldi, S. Chaotic Red Queen coevolution in a three species food chain. Proc. Roy. Soc. B 277, 2321-2330 (2012)

[17] de Visser, J. A. G. M., Elena, S. F. The evolution of sex: empirical insights into the roles of epistasis and drift. Nat. Rev. Genetics 8, 139-149 (2007)

[18] de Vladar, H.P., Santos, M. and Szathmáry, E., 2017. Grand views of evolution. Trends in Ecology and Evolution 32, 324-334.

[19] Dieckmann, U., Marrow, P., Law, R. Evolutionary cycling in predator-prey interactions: Population dynamics 
and the Red Queen. J. Theor. Biol. 176, 91-92 (1995)

[20] Drossel, B. Biological evolution and statistical physics. Adv. Phys. 50, 209-295 (2001)

[21] Duesberg, P. and Rasnick, D., 2000. Aneuploidy, the somatic mutation that makes cancer a species of its own. Cell motility and the cytoskeleton 47, 81-107.

[22] Dybdahl, M.F. and Storfer, A., 2003. Parasite local adaptation: red queen versus suicide king. Trends in Ecology and Evolution, 18,523-530.

[23] Ebert, D., 2008. Host-parasite coevolution: insights from the Daphnia-parasite model system. Current opinion in microbiology, 11,290-301.

[24] Ebner, M., 2006. Coevolution and the red queen effect shape virtual plants. Genetic Programming and Evolvable Machines 7, 103-123.

[25] Eldredge, N., and Gould, S. J. 1972. Punctuated equilibria: an alternative to phyletic gradualism. Models in paleobiology, pp.82-115.

[26] Fernandez, J.D., Lobo, D., Martn, G.M., Doursat, R. and Vico, F.J., 2012. Emergent diversity in an open-ended evolving virtual community. Artificial life 18, 199-222.

[27] Gould, S. J. The structure of evolutionary theory. Harvard University Press, Cambrigde, MA (2002)

[28] Hamilton, W. D. Sex vs. non-sex vs. parasite. Oikos 35, 282-290 (1980)

[29] Hoffman, A. Testing the Red Queen hypothesis. J. Evol. Biol. 4, 1-7 (1991)

[30] Ikegami, T., Kaneko, K. Evolution of host-parasitoid network through homeochaotic dynamics. Chaos 2, 397-407 (1992)

[31] Jaenike, J. An hypothesis to account for the maintenance of sex in populations. Evol. Theor. 3, 191-194 (1978)

[32] Kaneko, K., Ikegami, T. Homeochaos: dynamic stability of a symbiotic network with population dynamics and evolving mutation rates. Physica D 56, 406-429 (1992)

[33] Kauffman, S. A., Johnsen, J. Coevolution on the edge of chaos: Coupled fitness landscapes, poised states and coevolutionary avalanches. J. Theor. Biol. 149, 467-505 (1991)

[34] Liow, L.H., Van Valen, L. and Stenseth, N.C., 2011. Red Queen: from populations to taxa and communities. Trends Ecol. Evol. 26, 349-358.

[35] Marshall, C.R., 2017. Five palaeobiological laws needed to understand the evolution of the living biota. Nature Ecol. Evol. 1, 1-6.

[36] Morran, L. T., Schmidt, O. G., Gelarden, I. A., Parrish II, R. C., Lively, C. M. Running with the Red Queen: Host-parasite coevolution selects for biparental sex. Science 333, 216-218 (2011)

[37] Morris, J.J., Lenski, R.E. and Zinser, E.R., 2012. The Black Queen Hypothesis: evolution of dependencies through adaptive gene loss. MBio, 3(2).

[38] Newman, M. E. J., Palmer, R. G. Modeling Extinction:
Oxford University Press, New York.

[39] Ndung'u, T. and Weiss, R.A., 2012. On HIV diversity. AIDS 26, 1255-1260.

[40] Nolfi, S. and Floreano, D., 1998. Coevolving predator and prey robots: Do ?arms races? arise in artificial evolution?. Artificial life 4, 311-335.

[41] Quental, T.B. and Marshall, C.R., 2013. How the Red Queen drives terrestrial mammals to extinction. Science 341, 290-292.

[42] Quer, J., Huerta, R., Novella, I. S., Tsimring, L., Domingo, E., Holland, J. J. Reproducible nonlinear population dynamics and critical points during replicative competitions of RNA virus quasispecies. J. Mol. Biol. 264, 465-471 (1996)

[43] Rabajante, J.F., Tubay, J.M., Uehara, T., Morita, S., Ebert, D. and Yoshimura, J., 2015. Red Queen dynamics in multi-host and multi-parasite interaction system. Scientific Reports, 5,1-7.

[44] Raup, D.M. Biological extinction and Earth history. Science 231, 1528-1533 (1986)

[45] Robson, A.J., 2005. Complex evolutionary systems and the Red Queen. The Economic Journal, 115(504), pp.F211-F224.

[46] Roopnarine, P.D., Extinction cascades and catastrophe in ancient food webs. Paleobiology 32 (1), 1 (2006)

[47] Sardanyés, J., Solé, R. V. Red Queen strange attractors in host-parasite replicator gene-for-gene coevolution. Chaos, Solitons and Fractals 32, 1666-1678 (2007)

[48] Solé, R. V., Ferrer, R., González-García, Quer, J., Domingo, E. Red Queen dynamics, competition and critical points in a model of RNA virus quasispecies. J. theor. Biol. 198, 47-59 (1999)

[49] Solé, R. V., Bascompte, J., Manrubia, S. C. Extinction: bad genes or weak chaos? Proc. Roy. Soc. B 263, 161-168 (1996)

[50] Solé, R.V. and Sardanyés, J., 2014. Red Queen coevolution on fitness landscapes. In Recent Advances in the Theory and Application of Fitness Landscapes (pp. 301338). Springer, Berlin, Heidelberg.

[51] Stenseth, N. C., Maynard Smith, J. Coevolution in ecosystems: Red Queen evolution or stasis? Evolution 38, 870-880 (1984)

[52] Van Valen, L. Evolution as a zero-sum game for energy. Evol. Theory 4, 129-142 (1980)

[53] Van Valen, L. A new evolutionary law. Evol. Theory 1, 1-30 (1973)

[54] Van Valen L 1991 HeLa, a new microbial species. Evolutionary Theory 10, 71?74

[55] Van Der Laan, J.D. and Hogeweg, P., 1995. Predator?prey coevolution: interactions across different timescales. Proceedings of the Royal Society of London B 259, 35-42. 